\title{
Image Contrast Enhancement using Split Bregman Method *
}

\author{
Somayeh Gh. Bardeji, Isabel N. Figueiredo, Ercília Sousa \\ CMUC, Department of Mathematics, \\ University of Coimbra, Portugal.
}

\begin{abstract}
In this paper we propose a variational method for image contrast enhancement, by keeping the image details and correcting the non-uniform illumination. It is a minimization problem, where the objective functional consists of two different fitting terms: a $L^{1}$ term that matches the gradients of the input and reconstructed images, for preserving the image details, and a $L^{2}$ term that measures the misfit between the reconstructed image and the mean value of the input image, for reducing the image variance and thus correcting the illumination. For solving this minimization problem we apply the split Bregman method, which is an efficient and fast iterative method suitable for this type of non-differentiable and convex minimization problem, involving a $L^{1}$ term. Some experimental results show the effectiveness of the method.
\end{abstract}

\section{INTRODUCTION}

Image contrast enhancement is an image processing technique, whose purpose is to improve the image quality, for human interpretation of the image contents or for supplying a good input in automated image processing systems.

In the literature there exists a plethora of contrast enhancement methods, which are based, for example, on histogram equalization, edge enhancement, edge sharpening, filtering and restoration. For a detailed description of these type of methods see for example the book (Gonzalez \& Woods 2008). In this paper we focus on a particular variational PDE (partial differential equation) approach for contrast enhancement. We refer for example to the book (Aubert \& Kornprobst 2006) for an overview of the application of functional analysis techniques and the theory of partial differential equations to different image processing problems, such as restoration of degraded images, denoising, segmentation, inpainting, decomposition into cartoon and texture, optical flow and image classification.

In the variational approach, used herein, the objective is to minimize an appropriate energy (or functional), whose corresponding Euler-Lagrange equa-

*This work was partially supported by the research project PTDC/MATNAN/0593/2012 of FCT (the Portuguese national funding agency for science, research and technology) and by CMUC (the Centre for Mathematics of the University of Coimbra - UID/MAT/00324/2013), funded by the Portuguese Government through FCT/MEC and co-funded by the European Regional Development Fund through the Partnership Agreement PT2020. tion involves a PDE, that can be afterwards solved by a suitable computational method. Our energy functional is a modification of that proposed in (Morel, Petro, \& Sbert 2014). It is composed of two fitting terms. One fits the gradients of the input and reconstructed images, it is measured with the $L^{1}$-norm, and aims at preserving image details. The $L^{1}$-norm is the chosen measure because it has the property of better preserving discontinuities, when compared to the $L^{2}$ norm. The second term fits the reconstructed image with the mean value of the input image, in the $L^{2}$ norm, for correcting non-uniform illumination. Due to its particular structure, we then choose the split Bregman method (Goldstein \& Osher 2009) to solve this variational problem. This is a particular efficient iterative method applicable to a wide class of $L 1$ regularized optimization problems.

After this introduction the rest of the paper includes the description of the variational problem in Section 2 , its numerical solution in Section 3, some applications in Section 4 and finally the paper ends with some conclusions and comments.

\section{DESCRIPTION OF THE MODEL}

In (Morel, Petro, \& Sbert 2014) it is proposed the following variational model for contrast enhancement

$$
\begin{aligned}
& \min _{u}\left\{\int_{\Omega}|\nabla u-\nabla f|^{2} d x+\lambda \int_{\Omega}(u-\tilde{u})^{2} d x\right\} \\
& =\min _{u}\left\{\|\nabla u-\nabla f\|_{L^{2}(\Omega)}^{2}+\lambda\|u-\tilde{u}\|_{L^{2}(\Omega)}^{2}\right\},
\end{aligned}
$$


where $f: \Omega \rightarrow R$ is the original (grayscale) input image, $\Omega \subset R^{2}$ represents the image pixel domain, $x$ is a point (i.e. a pixel) in $\Omega, \nabla$ denotes the gradient operator, $|$.$| is the Euclidean norm in R^{2}, \tilde{u}$ is the mean value of the reconstructed image $u: \Omega \rightarrow R, L^{2}(\Omega)$ is the space of square integrable functions in $\Omega$, and $\|\cdot\|_{L^{2}(\Omega)}$ denotes the $L^{2}$-norm. This model contains two quadratic fitting terms. The first fits the gradients of $u$ and $f$, and consequently aims at preserving image details. The second term intends to reduce the effect of nonuniform illumination by fitting $u$ and its mean value $\tilde{u}$, thus by decreasing the image variance. The parameter $\lambda$ is a positive constant that balances the influence of the two fitting terms. After replacing $\tilde{u}$ by $\tilde{f}$, the mean value of the input image $f$, it is shown that the problem does not depend on the value $\tilde{f}$, and the problem is solved, for $\tilde{f}=0$ in the Fourier domain, using the discrete Fourier transform.

In this paper we modify the model (1) by replacing the $L^{2}$-norm by the $L^{1}$-norm for measuring the misfit between $\nabla u$ and $\nabla f$ and we also replace the mean value $\tilde{u}$ by $\tilde{f}$. This yields the following variational model

$$
\begin{aligned}
& \min _{u}\left\{\int_{\Omega}|\nabla u-\nabla f| d x+\frac{\lambda}{2} \int_{\Omega}(u-\tilde{f})^{2} d x\right\} \\
& =\min _{u}\left\{\|\nabla u-\nabla f\|_{L^{1}(\Omega)}+\frac{\lambda}{2}\|u-\tilde{f}\|_{L^{2}(\Omega)}^{2}\right\}
\end{aligned}
$$

where $L^{1}(\Omega)$ is the space of absolutely integrable functions in $\Omega$ and $\|\cdot\|_{L^{1}(\Omega)}$ denotes the $L^{1}$-norm. The reason for replacing the $L^{2}$-norm by the $L^{1}$-norm is related to the fact that the $L^{2}$-norm of the gradient tends to smear image discontinuities, as opposed to the $L^{1}$-norm that tends to preserve the discontinuities, which in image processing corresponds to sharp edges. In addition to this advantage, the presence of the $L^{1}$ fitting term in (2) permits the use of fast and effective algorithms for computing its solution. In effect, problem (2) belongs to the general class of $L^{1}$ regularized problems of the form

$\min _{u}\left\{\|\phi(u)\|_{L^{1}(\Omega)}+H(u)\right\}$

where both $\|\phi(u)\|_{L^{1}(\Omega)}$ and $H(u)$ are convex functions. This kind of models can be efficiently solved with the split Bregman method of (Goldstein \& Osher 2009). This is an appropriate algorithm for solving non-differentiable convex minimization problems, involving $L^{1}$ or $T V$ (total variation) terms. We refer to (Fang, Li, Zhang, \& Shen 2013, Getreuer 2012b, Goldstein, Bresson, \& Osher 2010, Yin, Osher, Goldfarb, \& Darbon 2008) for a few examples of different applications of the method.

In our case $\phi(u)=\nabla u-\nabla f$ and $H(u)=\lambda \| u-$ $\tilde{f} \|_{L^{2}(\Omega)}$, and in the next section we apply split Bregman method to solve (2).

\section{NUMERICAL SOLUTION BASED ON SPLIT BREGMAN METHOD}

A critical and first aspect of the split Bregman method is the separation of the $L^{1}$ and $L^{2}$ terms, which is achieved by introducing an auxiliary variable. Thus, we first replace (2) by the following constrained optimization problem

$$
\begin{aligned}
& \min _{u}\left\{\|d\|_{L^{1}(\Omega)}+\frac{\lambda}{2}\|u-\tilde{f}\|_{L^{2}(\Omega)}\right\} \\
& \text { subject to } \quad d=\nabla u-\nabla f,
\end{aligned}
$$

and then reformulate it as an unconstrained problem, by introducing a quadratic penalty function, that is

$$
\begin{aligned}
& \min _{u}\left\{\|d\|_{L^{1}(\Omega)}+\frac{\lambda}{2}\|u-\tilde{f}\|_{L^{2}(\Omega)}^{2}\right. \\
&\left.+\frac{\alpha}{2}\|d-(\nabla u-\nabla f)\|_{L^{2}(\Omega)}^{2}\right\} .
\end{aligned}
$$

Then, the split Bregman method consists in solving the following sequence of problems for $k=0,1,2, \ldots$.

$$
\left\{\begin{array}{l}
\left(u^{k+1}, d^{k+1}\right)= \\
\arg \min _{d, u}\left\{\|d\|_{L^{1}(\Omega)}+\frac{\lambda}{2}\|u-\tilde{f}\|_{L^{2}(\Omega)}^{2}\right. \\
\left.\quad+\frac{\alpha}{2}\left\|d-(\nabla u-\nabla f)-b^{k}\right\|_{L^{2}(\Omega)}^{2}\right\}, \\
b^{k+1}=b^{k}+\nabla u^{k+1}-\nabla f-d^{k+1} .
\end{array}\right.
$$

where the new vector $b^{k}$ results from the Bregman iteration (Bregman 1967), that is a strategy for enforcing the constraint $d=\nabla u-\nabla f$, using a fixed penalty parameter $\alpha$. This strategy is an alternative to the conventional continuation technique to solve (3) with an increasing sequence of penalty parameters $\alpha_{1}<\alpha_{2}<\ldots<\alpha_{n}$ tending to $\infty$, for accurately enforcing the constraint. The minimization problem in (4) is solved by iteratively minimizing with respect $u$ and $d$, alternatively, which means the following two steps are performed.

Step 1- Minimization with respect to $u$ (with $d$ fixed)

$$
\begin{aligned}
u^{k+1}=\arg \min _{u}\left\{\frac{\lambda}{2}\|u-\tilde{f}\|_{L^{2}(\Omega)}^{2}\right. & \\
+ & \left.\frac{\alpha}{2}\left\|d^{k}-(\nabla u-\nabla f)-b^{k}\right\|_{L^{2}(\Omega)}^{2}\right\}
\end{aligned}
$$

for which the optimality condition (derived from the Euler-Lagrange equation) is, in $\Omega$

$$
(\lambda-\alpha \Delta) u^{k+1}=\lambda \tilde{f}-\alpha \operatorname{div}\left(d^{k}+\nabla f-b^{k}\right),
$$

with $\Delta$ and div denoting the Laplace and divergence operators, respectively, along with the nonhomogeneous Neumann boundary condition on the boundary $\partial \Omega$ of $\Omega$

$$
\frac{\partial u^{k+1}}{\partial n}=\left(d^{k}-\nabla f-b^{k}\right) \cdot n,
$$


where "." denotes the inner product in $R^{2}$ and $n$ is the unit outward normal to $\partial \Omega$.

This problem can be solved efficiently with the Gauss-Seidel method, since the system is diagonally dominant. The solution $u_{i, j}^{k+1}$ at each pixel $(i, j)$ in $\Omega$ (excepting in $\partial \Omega$ ) is defined by

$$
\begin{aligned}
& u_{i, j}^{k+1}=\frac{1}{\lambda+4 \alpha}\left[\alpha U_{i, j}+\lambda \tilde{f}_{i, j}-\alpha v_{i, j}^{k}\right] \\
& U=\left(U_{i, j}\right):=\left(u_{i-1, j}^{k+1}+u_{i+1, j}^{k}+u_{i, j-1}^{k+1}+u_{i, j+1}^{k}\right) \\
& v^{k}=\left(v_{i, j}^{k}\right):=\left(\operatorname{div}\left(d^{k}+\nabla f-b^{k}\right)\right)_{i, j} .
\end{aligned}
$$

Here we use finite differences for approximating the derivatives in the gradient $\nabla$ and divergence div operators, respectively. In particular the discretization used for $v_{i, j}^{k}$ is obtained by applying backward finite differences for $\operatorname{div} d^{k}$ and $\operatorname{div} b^{k}$ and centered finite differences for $\Delta f$

$$
\begin{array}{r}
v_{i, j}^{k}=2 d_{i, j}^{k}-d_{i-1, j}^{k}-d_{i, j-1}^{k}-2 b_{i, j}^{k}+b_{i-1, j}^{k}+b_{i, j-1}^{k} \\
+f_{i-1, j}^{k}+f_{i+1, j}^{k}+f_{i, j-1}^{k}+f_{i, j+1}^{k}-4 f_{i, j}^{k} .
\end{array}
$$

The Neumann boundary condition is implicitly imposed in $\partial \Omega$, the boundary of the rectangular pixel domain, by using backward finite differences, in the right and top sides, and forward finite differences in the left and bottom sides.

Step 2- Minimization with respect to $d$ (with $u$ fixed)

$$
\begin{aligned}
& d^{k+1}=\arg \min _{d}\left\{\|d\|_{L^{1}(\Omega)}\right. \\
&\left.+\frac{\alpha}{2}\left\|d-\left(\nabla u^{k+1}-\nabla f\right)-b^{k}\right\|_{L^{2}(\Omega)}^{2}\right\} .
\end{aligned}
$$

This problem can be explicitly solved using shrinkage operation (known as well as soft thresholding) at each pixel $(i, j)$

$d_{i, j}^{k+1}=\operatorname{shrink}\left(\left(\nabla u^{k+1}-\nabla f+b^{k}\right)_{i, j}, \frac{1}{\alpha}\right)$

where for $z, \gamma \in R$

$\operatorname{shrink}(z, \gamma)=\frac{z}{|z|} \times \max (|z|-\gamma, 0)$.

Summarizing, the split Bregman method for model (2) is as follows:

\section{Algorithm -}

Input - Original image $f$.

Initialize - $u^{0}=f, d^{0}=b^{0}=0$, and fix $\lambda, \alpha$, tol.

While $\left|u^{k}-u^{k-1}\right|>$ tol

$u^{k+1}=\frac{1}{\lambda+4 \alpha}\left[\alpha U+\lambda \tilde{f}-\alpha v^{k}\right], \quad$ in $\Omega$,

$\frac{\partial u^{k+1}}{\partial n}=\left(d^{k}-\nabla f-b^{k}\right) \cdot n, \quad$ in $\partial \Omega$,

$d^{k+1}=\operatorname{shrink}\left(\nabla u^{k+1}-\nabla f+b^{k}, \frac{1}{\alpha}\right)$,

$b^{k+1}=b^{k}+\nabla u^{k+1}-\nabla f-d^{k+1}$.

\section{End}

Output - Image $u^{k}$.

\section{APPLICATIONS}

Some results obtained with our proposed model are shown in this section. All the experiments were implemented with the software MATLAB B R2014a (The Mathworks, Inc.)

Figure 1 shows the contrast enhancement with our method for a standard test image (a scalar image with $512 \times 343$ pixels), downloaded from the IPOL archive (http://www.ipol.im/). As this figure demonstrates, the details are kept and the dark regions become more visible in the enhanced image. In addition, and as expected, when $\lambda$ increases ( $\lambda$ is the parameter associated with the fitting term intended to reduce the non-uniform illumination) the result tends to the mean value of the input image.
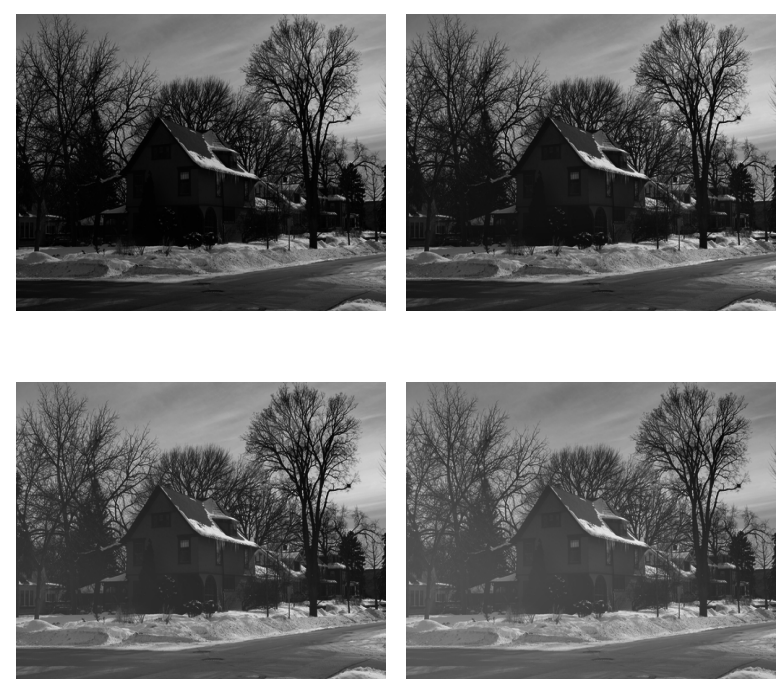

Figure 1: Top left: Original image. Top right: $\lambda=0.005, \alpha=1$. Bottom left: $\lambda=0.01, \alpha=1$. Bottom right: $\lambda=0.05, \alpha=1$.

Figure 2 depicts the results of our method applied to a medical (RGB - red, green, blue) image (with $536 \times 536$ pixels), acquired with the wireless capsule Pillcam Colon 2 of Given Imaging. It displays a colonic polyp (the reddish region in the top left subfigure) exhibiting strong texture. We applied the algorithm independently to each channel. The original medical image (the top left subfigure) has a nonuniform illumination, with low contrast in some regions, that is corrected and enhanced with the proposed method. The influence of the model parameters $(\lambda$ and $\alpha$ ) is also illustrated in these results. Increasing $\lambda$ results in an averaged image, tending to the mean value of the input image, and by increasing $\alpha$ the contrast enhancement is enforced.

In Figure 3 we can see the results for another medical image (with size $536 \times 536$ pixels). It is an inhomogeneous illuminated retinal fundus image, provided by the company Retmarker (http://www.retmarker.com/), and obtained from a patient screened according to the Diabetic Retinopathy Screening Program of Portugal. We have processed with our method the grayscale version (sec- 



Figure 2: Top left: Polyp image obtained with Pillcam Colon 2, by courtesy of University Hospital of Coimbra, Portugal. Top right: $\lambda=1, \alpha=150$. Bottom left: $\lambda=1, \alpha=200$. Bottom right: $\lambda=2, \alpha=200$.


Figure 3: First column: Original (RGB) retinal fundus image and results with $(\lambda=1, \alpha=5)$ in 2 nd row and $(\lambda=5, \alpha=5)$ in 3rd row. Second column: Grayscale version and results with $(\lambda=1$, $\alpha=5)$ in 2 nd row and $(\lambda=5, \alpha=5)$ in 3rd row.

ond column) as well as each color channel separately (first column). Again these results show the good contrast enhancement improvement achieved with our method.

Figure 4 shows the result of the proposed methodology for a MRI image, downloaded from the IPOL archive (http://www.ipol.im/), and also a comparison to the result obtained with the online demo of (Morel, Petro, \& Sbert 2014). We remark that our method operates directly on the input image (exhibiting intensity inhomogeneities) whereas the displayed result with the technique of (Morel, Petro, \& Sbert 2014) involve pre and post processing steps that consist on the application of a simplest color balance technique (Limare, Lisani, Morel, Petro, \& Sbert 2011). We can notice that our method performs slightly better in the dark down region on the left part of the image. We also emphasize that our method doesn't use any pre or post processing methodology.
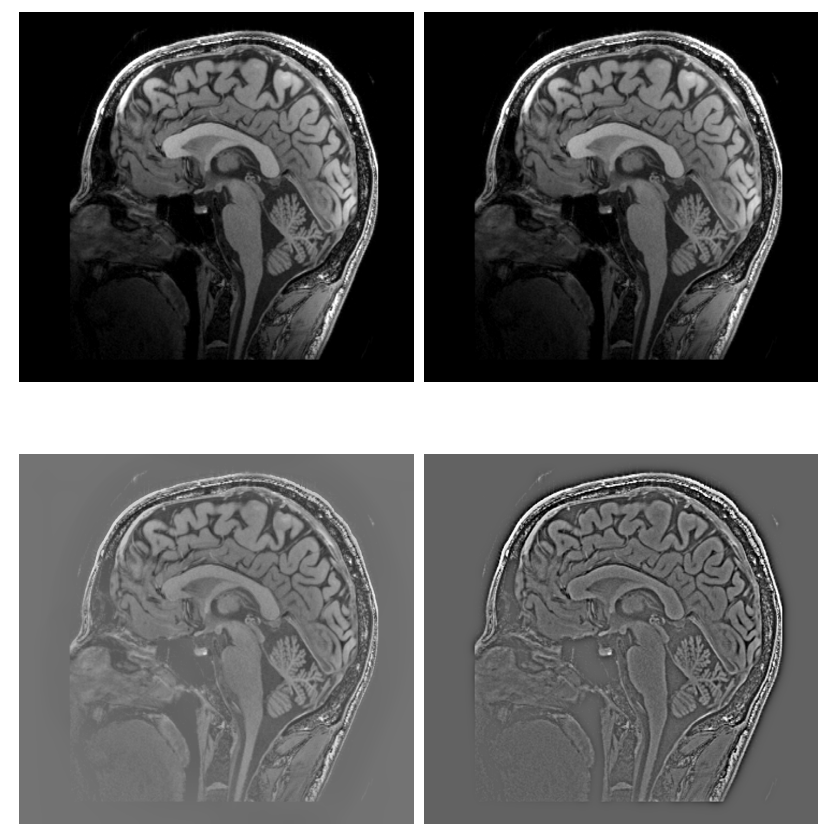

Figure 4: Top left: Original MRI and input for the proposed method. Top right: Original MRI with $2 \%$ saturation and input for the model solved with the screened Poisson equation (Morel, Petro, \& Sbert 2014). Bottom left: Result for the proposed method with $(\lambda=1, \alpha=10)$. Bottom right: Result applying a simplest color balance with $2 \%$ saturation to the solution obtained with the screened Poisson equation and with $\lambda=0.05$.

\section{CONCLUSIONS}

In this paper we propose an inverse variational model for image contrast enhancement. It is a modification of the screened Poisson equation for image contrast enhancement as proposed by (Morel, Petro, \& Sbert 2014). The difference with respect to this latter model is in the term that matches the gradients of the input and reconstructed images, that is now measured in the $L^{1}$-norm (instead of the $L^{2}$-norm as in (Morel, Petro, $\&$ Sbert 2014)), because the former has the advantage of better preserving edges in image processing applications. The presence of this $L^{1}$-norm term enables 
the proposed model to be solved by the split Bregman technique, which is an efficient procedure that has the advantage of not requiring regularization, continuation, or the enforcement of inequality constraints.

Applications of the proposed method to different types of images show its good performance. We also remark that our methodology does not include pre and post processing steps as it happens in the methodology presented in (Morel, Petro, \& Sbert 2014), where the best reconstructed images have been obtained with pre and post processing steps, involving the application of a simplest color balance technique (Limare, Lisani, Morel, Petro, \& Sbert 2011) applied before and after solving the model with the screened Poisson equation.

The model we propose involves some parameters that are tuned and fixed manually. In the future an automatic or self-adapting method for choosing these parameters will be studied. Moreover an additional analysis concerning the comparison of the results obtained with the present method with those obtained with the original model in (Morel, Petro, \& Sbert 2014) will be performed, as well as a comparison with the ACE method (Getreuer 2012a), which is one of the most effective contrast enhancement methods.

\section{REFERENCES}

Aubert, G. \& P. Kornprobst (2006). Mathematical problems in image processing: partial differential equations and the calculus of variations, Volume 147. Springer Science \& Business Media.

Bregman, L. M. (1967). The relaxation method of finding the common point of convex sets and its application to the solution of problems in convex programming. USSR Computational Mathematics and Mathematical Physics 7(3), 200-217.

Fang, F., F. Li, G. Zhang, \& C. Shen (2013). A variational method for multisource remote-sensing image fusion. International Journal of Remote Sensing 34(7), 24702486.

Getreuer, P. (2012a). Automatic color enhancement (ace) and its fast implementation. Image Processing On Line (2012).

Getreuer, P. (2012b). Rudin-Osher-Fatemi total variation denoising using split Bregman. Image Processing On Line 10.

Goldstein, T., X. Bresson, \& S. Osher (2010). Geometric applications of the split bregman method: segmentation and surface reconstruction. Journal of Scientific Computing 45(1-3), 272-293.

Goldstein, T. \& S. Osher (2009). The Split Bregman method for L1-regularized problems. SIAM Journal on Imaging Sciences 2(2), 323-343.

Gonzalez, R. C. \& R. E. Woods (2008). Digital Image Processing (3rd Edition). NJ, USA: Prentice-Hall, Inc.

Limare, N., J.-L. Lisani, J.-M. Morel, A. B. Petro, \& C. Sbert (2011). Simplest color balance. Image Processing On Line 1.

Morel, J.-M., A.-B. Petro, \& C. Sbert (2014). Screened Poisson equation for image contrast enhancement. Im- age Processing On Line 4, 16-29.

Yin, W., S. Osher, D. Goldfarb, \& J. Darbon (2008). Bregman iterative algorithms for L1-minimization with applications to compressed sensing. SIAM Journal on Imaging Sciences 1(1), 143-168. 